Research Paper

\title{
Migration of mesenchymal stem cells to tumor xenograft models and in vitro drug delivery by doxorubicin
}

Senthilkumar Kalimuthu, Liya Zhu, Ji Min Oh, Prakash Gangadaran, Ho Won Lee, Se hwan Baek, Ramya Lakshmi Rajendran, Arunnehru Gopal, Shin Young Jeong, Sang-Woo Lee, Jaetae Lee and Byeong-Cheol $\mathrm{Ahn}^{凶}$

Department of Nuclear Medicine, School of Medicine, Kyungpook National University, Kyungpook National University Hospital, Daegu, Republic of Korea

$\triangle$ Corresponding author: Prof. Byeong-Cheol Ahn., M.D., Ph.D., Department of Nuclear Medicine, School of Medicine, Kyungpook National University, Kyungpook National University Hospital, 50, Samduk 2-ga, Jung Gu, Daegu-700-721, Republic of Korea. Tel: 82-53-420-5583; Fax: 82-53-422-0864; Email: abc2000@knu.ac.kr

(C) Ivyspring International Publisher. This is an open access article distributed under the terms of the Creative Commons Attribution (CC BY-NC) license (https://creativecommons.org/licenses/by-nc/4.0/). See http://ivyspring.com/terms for full terms and conditions.

Received: 2018.02.26; Accepted: 2018.06.01; Published: 2018.06.22

\begin{abstract}
Mesenchymal stem cells (MSCs) show therapeutic effects in various types of diseases. MSCs have been shown to migrate towards inflamed or cancerous tissues, and visualized after sacrificing the animal. MSCs are able to deliver drugs to target cells, and are an ideal candidate for cancer therapy. The purpose of this study was to track the migration of MSCs in tumor-bearing mice; MSCs were also used as drug delivery vehicles. Human breast cancer cells (MDA-MB-231) and anaplastic thyroid cancer cells (CAL62) were transduced with lentiviral particles, to express the Renilla luciferase and mCherry (mCherry-Rluc) reporter genes. Human bone marrow-derived MSCs were transduced with lentiviral particles, to express the firefly luciferase and enhanced green fluorescence protein (Fluc2-eGFP) reporter genes (MSC/Fluc). Luciferase activity of the transduced cells was measured by bioluminescence imaging (BLI). Further in vitro migration assays were performed to confirm cancer cells conditioned medium dependent MSC and doxorubicin (DOX) treated MSC migration. MSCs were loaded with DOX, and their therapeutic effects against the cancer cells were studied in vitro. In vivo MSC/Fluc migration in mice having thyroid or breast cancer xenografts was evaluated after systemic injection. Rluc activity of CAL62/Rluc $\left(R^{2}=0.911\right)$, MDA-MB-231/Rluc $\left(R^{2}=0.934\right)$ cells and Fluc activity of MSC/Fluc $\left(R^{2}=0.91\right)$ cells increased with increasing cell numbers, as seen by BLI. eGFP expression of MSC/Fluc was confirmed by confocal microscopy. Similar migration potential was observed between MSC/Fluc and naïve MSCs in migration assay. DOX treated MSCs migration was not decreased compared than MSCs. Migration of the systemically injected MSC/Fluc cells into tumor xenografts (thyroid and breast cancer) was visualized in animal models $(p<0.05)$ and confirmed by ex vivo $(p<0.05)$ BLI. Additionally, MSCs delivered DOX to CAL62/Rluc and MDA-MB-231/Rluc cells, thereby decreasing their Rluc activities. In this study, we confirmed the migration of MSCs to tumor sites in cancer xenograft models using both in vivo and ex vivo BLI imaging. DOX-pretreated MSCs showed enhanced cytotoxic effects. Therefore, this noninvasive reporter gene (Fluc2)-based BLI may be useful for visualizing in vivo tracking of MSCs, which can be used as a drug delivery vehicle for cancer therapy.
\end{abstract}

Key words: Human mesenchymal stem cells, Breast cancer, Anaplastic thyroid cancer, CAL62 cells, bioluminescent imaging

\section{Introduction}

Mesenchymal stem cells (MSCs) are multipotent and self-renewing progenitor cells that can differentiate into multiple mesoderm lineages. MSCs have notable tropic and immunosuppressive characteristics in injured tissues [1-4]. Owing to their migration capacity, MSCs could be considered as clinically relevant cell types for various diseases; they may also serve as a potential type of therapeutic cells. Clinical studies on MSCs have increased in the past 20 years [2] because of the convenient expansion capability of these cells; MSCs have been used for treating spinal cord injuries $[5,6]$, cardiac regener- 
ation therapy [7], muscular dystrophy [8], myocardial infarction [9, 10], graft-versus-host-disease [11], and cancers [12-14].

After the systemic intravenous injection of MSCs and their subsequent localization, the experimental animals were sacrificed; this was followed by fluorescent visualization [15], immunohistochemistry $[16]$, or DNA-PCR $[17,18]$. MSC homing to tumor and diseases model were studied with different methods such as optical and non-optical methods [4]. Kidd et al., reported that MSC isolated from human bone marrow and their localization was confirmed in MDA-MB-231 lung metastasis mice after systemic injection at day 29 in lung and liver and they also shown that tumor tropism of mouse MSC in subcutaneously established $4 \mathrm{~T} 1$ breast tumor at 0.5, 6 and 12 day using bioluminescence imaging (BLI) [1].

Molecular imaging strategies can visualize the fate of cells non-invasively by in vivo serial imaging acquisition without animal sacrifice, and has been an invaluable tool for developing cell-based therapeutic strategies [19]. Reporter genes can be passed on to the progeny, making this a better approach for viewing transplanted cells in vivo $[20,21]$. Renilla luciferase (Rluc) or firefly luciferase (Fluc) reporter gene was used for noninvasive BLI [3, 16, 22-24]. BLI measures the light emitted from cells labeled with luminescent enzymes (e.g., luciferase), react with their substrate and produce the light $[2,25]$.

The major objective of cancer chemotherapies is to concentrate the drugs that can kill cancer cells into the tumor microenvironment with less collateral toxicity [26]. Enhanced cancer targeting with technical approaches such as immunoconjugates with specific tumor antigen [27], nanoparticles [28], or manipulated stem cells [29], has been developed; these methods prove to be good choices for delivering cytotoxic agents. Therefore, in this study, we aimed to confirm the migration potency of MSCs to tumors and whether Doxorubicin (DOX)-primed MSCs have cytotoxic effects on cancer cells. Importance of our study is showing MSC migration to thyroid tumor xenograft, there was no direct evidence tumor tropism of MSC in thyroid tumor model, and also demonstrating migration of MSC to breast cancer in MDA-MB-231 tumor xenograft mouse model by optical molecular imaging, and the possible drug delivery-based in vitro therapeutic effects of DOX-primed MSCs against breast and thyroid cancer.

\section{Material and Methods}

\section{Cell culture}

DMEM-F12 and DMEM-High were obtained from Hyclone (Logan, UT, USA). Antibiotics were obtained from Gibco-Invitrogen (Carlsbad, CA, USA). Human adult bone marrow-derived MSCs (hMSCs) were purchased from ATCC (Manassas, VA, USA) and it was isolated from bone marrow, received at the second passage number (P2) with characteristics of differentiation potential (Cat No: ATCC-PSC-500012). MSCs were grown in DMEM-F12 containing $10 \%$ fetal bovine serum and the antibiotic gentamicin (Gibco, Invitrogen), and maintained in a humidified incubator at $37^{\circ} \mathrm{C}$ with $5 \% \mathrm{CO}_{2}$. MDA-MB-231 cells were purchased from ATCC, and CAL62 (an anaplastic thyroid cancer cell line) was purchased from DSMZ-Germany (Braunschweig, Germany). Both cell types were grown in DMEM supplemented with $10 \%$ FBS and a 1\% penicillin/streptomycin solution (HyClone). We used viral vectors under the bio safety cabinet with institutional safety procedure.

\section{Lentiviral transduction of MSCs}

MSCs were transduced with lentiviral particles containing the CMV promoter (GeneCopoeia, Rockville, MD, USA), to express firefly luciferase and green fluorescent protein (eGFP-Fluc); the cells were incubated overnight with a solution containing the lentiviral particles and polybrene $(8 \mu \mathrm{g} / \mathrm{mL}$ ). eGFP-positive MSC cells were sorted by a FACS Aria III cell sorter (BD Biosciences, Franklin Lakes, NJ, USA), and the separated cells were named as hMSC/Fluc. Fluc activity in the MSC/Fluc cells was measured by BLI with an IVIS lumina II (Caliper Life Sciences, Hopkinton, MA, USA) by adding D-luciferin as a substrate $(150 \mu \mathrm{g} / \mathrm{ml})$. After lentiviral transduction, MSC/Fluc cells were generated and used for the present study with passage number 8 (P8).

\section{Lentiviral transduction of cancer cells}

MDA-MB-231 and CAL62 cells were transduced with lentiviral particles containing the CMV promoter (GeneCopoeia), to express Renilla luciferase and mCherry protein (mCherry-Rluc). Transduced cells were prepared according to our previous studies [30, 31]. The generated stable cell lines were named as MDA-MB-231/Rluc and the CAL62/Rluc. Rluc activity of transduced cells was measured with the IVIS lumina II by adding coelentrazine $(10 \mu \mathrm{g} / \mathrm{ml})$ as a substrate.

\section{Confocal microscopy analysis}

MSC/Fluc and naïve MSCs cells $\left(5 \times 10^{5}\right)$ were seeded into an 8-well cell plate. Twenty-four hours after plating, the medium was removed and washed with phosphate buffered saline (PBS). Next, the cells were fixed with $4 \%$ paraformaldehyde for $10 \mathrm{~min}$ and then washed with PBS. The slides were then mounted with DAPI mounting medium (Vector Laboratories, Burlingame, CA, USA). eGFP images were analyzed 
by confocal laser microscopy (LSM 5 exciter; Zeiss, Oberkochen, Germany).

\section{Conditioned medium collection from cancer cells}

CAL62 and MDA-MB-231 cells were grown in $100 \mathrm{~mm}$ culture petri dish and after it reached $70 \%$ confluency the medium was removed and washed with PBS, then added fresh $10 \mathrm{ml}$ SFM for $24 \mathrm{~h}$. After $24 \mathrm{~h}$, collected conditioned medium (CM) was centrifuged to remove the cell debris and filtered through $0.45 \mu \mathrm{m}$ syringe filter and stored in $-20^{\circ}$ until used for experiments.

\section{In vitro migration assay}

To confirm the functional ability of the transduced MSCs, we performed in vitro migration assays with 8 - $\mu \mathrm{m}$ pores (Corning Costar, Cambridge, MA, USA), according to our previous study [31]. $1 \times$ $10^{5}$ MSC and MSC/Fluc cells were mixed in $500 \mu \mathrm{L}$ of serum-free medium and added to the upper chamber of the Transwell migration chamber; the bottom chamber contained $0.5 \%$ fetal bovine serum (FBS) or CAL62 and MDA-MB-231 cells CM. After a 4-h incubation at $37^{\circ} \mathrm{C}$ and $5 \% \mathrm{CO}_{2}$, the lower surface of the migrated cells containing the membrane was fixed with $4 \%$ paraformaldehyde and stained with $0.1 \%$ crystal violet. A phase contrast microscope was used to count the migrated cell numbers in three random fields. The counted cells were plotted as a graph of cells migrated per field.

\section{In vitro therapeutic effect of MSCs}

In order to confirm the therapeutic effect of MSCs, we pretreated MSCs with DOX $(5 \mu \mathrm{M})$ overnight. After a 12-h incubation, the MSCs were washed 3 times with PBS and trypsinized. We then cultured CAL62/Rluc and MDA-MB-231/Rluc cells (1x10 cells) with different ratios (1:0.5, 1:1 and 1:2) of naïve MSCs or DOX-pretreated MSCs for 24 and $48 \mathrm{~h}$. The Rluc activity of CAL62/Rluc and MDA-MB-231/ Rluc was measured after 24 and $48 \mathrm{~h}$ by IVIS imaging with the addition of coelentrazine as a substrate. After measurement, the region of interest (ROI) was drawn individually in each well and the signal intensity of each ROI was measured. The emitted signal was expressed as photons/second ( $\mathrm{p} / \mathrm{s})$.

\section{Effect DOX on MSC cell viability by CCK-8}

To confirm the effects of DOX on cell viability of MSCs, cells were seeded in the 96-well plates $\left(5 \times 10^{3} /\right.$ well $)$ and give different concentration of DOX $(1.25,2.5,5,10$ and $20 \mu \mathrm{M})$ for $24 \mathrm{~h}$. Cell viability was assessed after $24 \mathrm{~h}$ using Cell Counting Kit-8 (CCK-8) (Dojindo, Kumamoto, Japan).

\section{In vivo MSC migration in breast and thyroid cancer}

MDA-MB-231/Rluc breast cancer tumor xenografts were established by injecting $5 \times 10^{6}$ MDA-MB-231 cells mixed with matrigel (1:4 dilution) into the right flank of 6-week-old female nude mice (BALB-c/nu). The animal experiments were approved by the Institutional Animal Care and Use Committee. One month after the inoculation with MDAMB-231/Rluc cells, tumor growth was assessed by measuring the Rluc activity using BLI. To prepare CAL62/Rluc tumor xenografts, $5 \times 10^{6}$ CAL62/Rluc cells mixed with matrigel (1:4 dilution) and injected right flank of the nude mice. Rluc activity was confirmed by IVIS after 3 months. Mice with MDA-MB-231/Rluc or CAL62/Rluc xenografts received $1 \times 10^{6} \mathrm{MSC} /$ Fluc cells via intravenous injection (i.v.). The in vivo migration of MSC/Fluc was visualized by BLI. We performed separate experiments for MDA-MB-231/Rluc and CAL62/ Rluc. For each xenograft model, we used three mice per group namely (1) control and (2) MSC/Fluc group. To image the Fluc activity of MSC/Fluc, each mouse was injected with D-luciferin at $150 \mathrm{mg} / \mathrm{kg}$ body weight $(100 \mu \mathrm{L})$ via intraperitoneal injection (i.p.). Oneminute exposure images were acquired with medium binning. Optical images of the migrated MSC/Fluc cells obtained from IVIS were displayed and analyzed with Living Image Software. BLI signals were quantitatively measured by drawing ROIs manually in the tumor area of the mice to quantify the signal intensity and emitted signal intensity, which was represented as $\mathrm{p} / \mathrm{s}$. For tumor Rluc activity, coelenterazine in PBS was i.v. injected and images were immediately acquired by IVIS.

\section{Ex vivo Fluc activity in tumors}

MSC/Fluc activity was measured after $24 \mathrm{~h}$ cell injection and then CAL62/Rluc and MDA-MB-231/ Rluc tumors were excised and kept in a 24 well plate with $500 \mu \mathrm{l}$ of PBS, then added D-luciferin as a substrate and measured the Fluc activity immediately by IVIS. The signal intensity was represented as $\mathrm{p} / \mathrm{s}$.

\section{Immunohistochemistry analysis for GFP}

In order to confirm the migrated MSC/Fluc cells in the tumor region, $10 \%$ formalin fixed tissues were embedded in paraffin. $5 \mu \mathrm{m}$ paraffin sections were subjected with anti-GFP antibody (Millipore, USA) and stained with a DAB (3,3'Diaminobenzidine) kit. The positive staining was taken photograph under a light microscope (40x magnifications).

\section{Statistical analysis}

Experiments were performed in triplicate for in vitro studies and three mice were used for in vivo 
analysis. Data were expressed as the means \pm standard deviation (SD) and a $p$-value $<0.05$ was considered statistically significant, according to the Student's $t$-test.

\section{Results}

\section{Characterization of MSC/Fluc cells}

MSC/Fluc cells were prepared by lentiviral transduction. Fluc activity of MSC/Fluc was measured by BLI, which increased with increasing cell numbers (Figure 1A, $R^{2}=0$ 91). eGFP was assessed by confocal microscopy (Figure 1B).

\section{Cancer cell Rluc activity}

For visualizing the tumor growth, MDA-MB-231 and CAL62 cancer cells were successfully transduced with lentiviral particles with the dual-reporter gene (mCherry-Rluc) was driven by a constitutive CMV promoter. The Rluc activity of MDA-MB-231/Rluc (Figure 1C, $R^{2}=0$ 911) and CAL62/Rluc (Figure 1D, $R^{2}=0$ 934) cells increased with increasing cell numbers, as confirmed by BLI. No significant change was noted in proliferation rate between parental and transduced cells (data not shown).

\section{In vitro migration of MSCs}

We performed transwell assay to monitor the migration of the transduced MSC/Fluc cells with chemoattractant $(0.5 \% \mathrm{FBS})$ or conditioned medium of cancer cells. A similar number of both MSCs and MSC/Fluc cells migrated towards the chemoattractant (Figure 2A). This result supports the fact that the retroviral transduction does not hamper the functionnal ability of the MSCs. MSCs showed endogenous tropism to MDA-MB-231 and CAL62 cancer cells conditioned medium was significantly $(p<0.001)$ higher compared than those with SFM (Figure 2B). Also the DOX MSC treated cells has no significant changes between MSC/Fluc cells migration (Figure 2B) with respective of cancer cells CM. The CAL62 $\mathrm{CM}$ dependent migration was significantly $(p<0.05)$ increased compared than MDA-MB-231 CM.
A
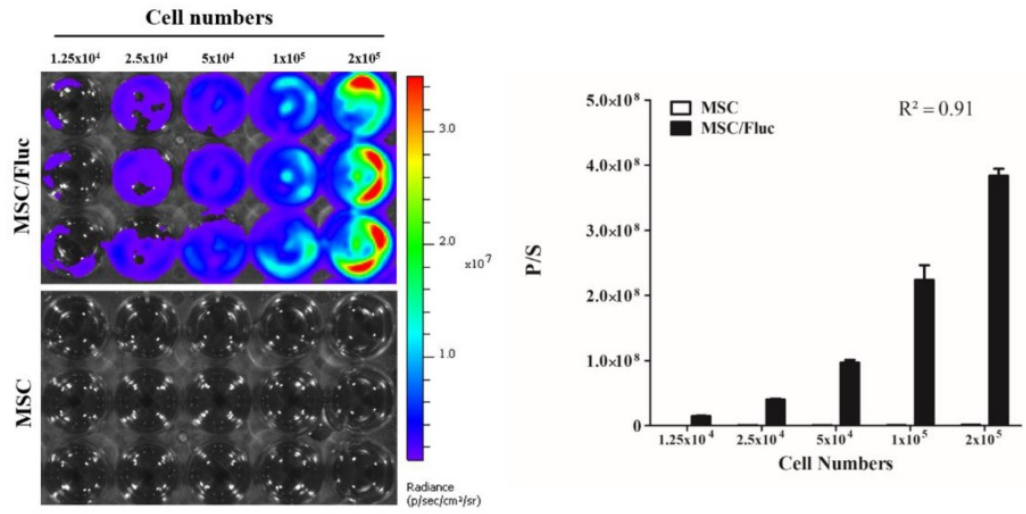

B
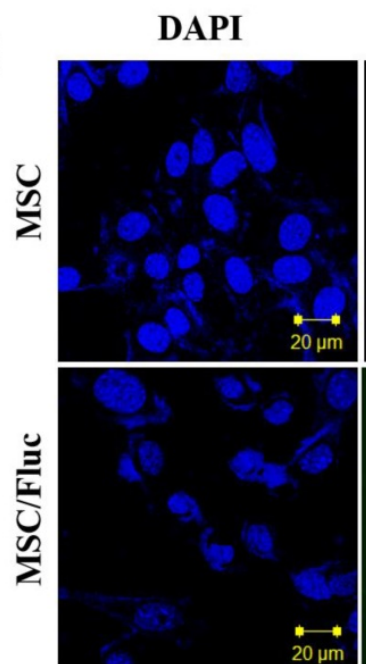

eGFP
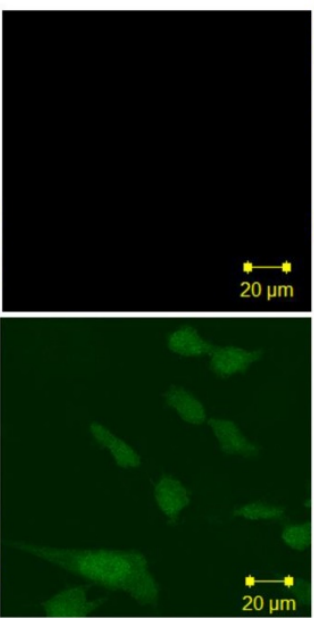
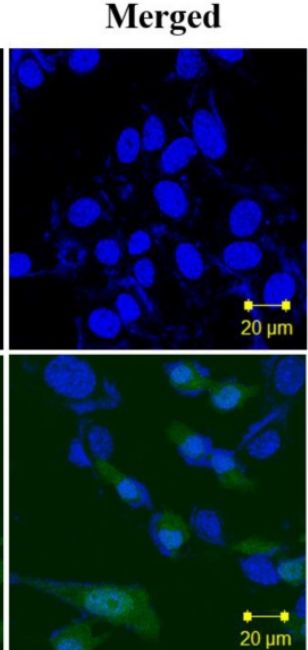

C
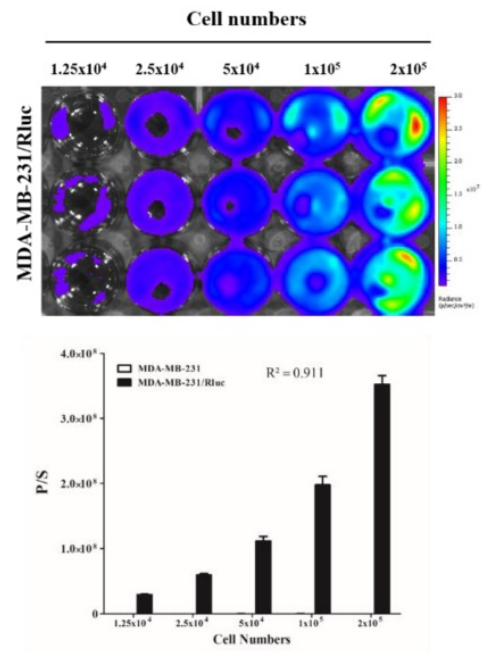

$\mathbf{D}$
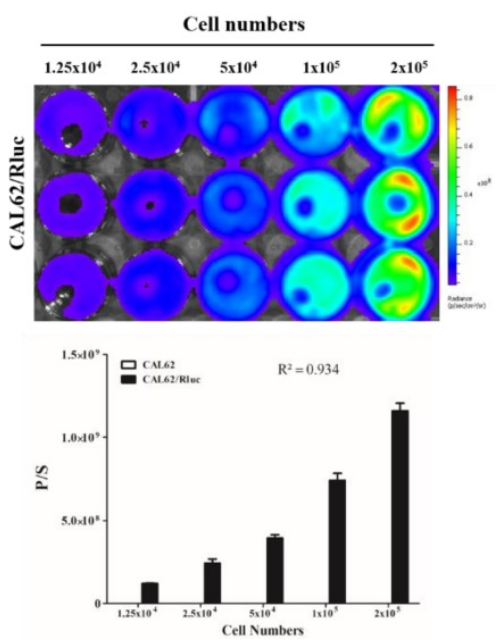

Figure 1. Characterization of MSC/Fluc and cancer cells. An increasing number of cells were plated and their luciferase activities were measured by BLI after $24 \mathrm{~h}$ of plating. A) Fluc activity and quantitative measurement of MSC/Fluc cells. B) Confocal microscopy image of eGFP in transduced MSC/Fluc cells. C) Rluc activity of the anaplastic thyroid cancer cells (CAL62/Rluc). D) Rluc activity of the breast cancer cells (MDA-MB-231/Rluc). Data were expressed as the means \pm standard deviation (SD). 
A
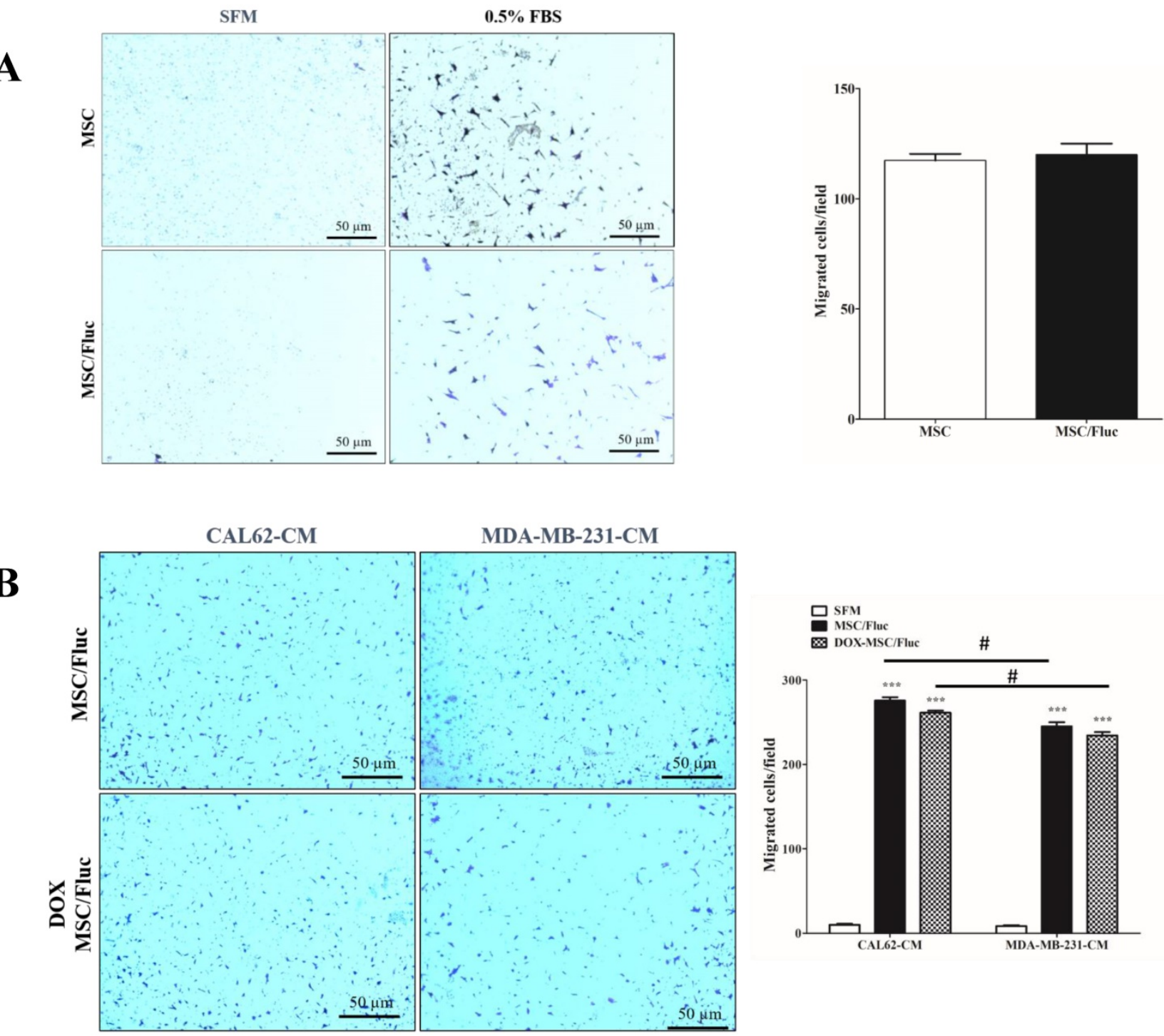

Figure 2. In vitro migrations of MSCs. MSCs and MSC/Fluc cells were mixed with serum-free media (SFM) and placed in the upper chamber, while the bottom chamber contained $0.5 \% \mathrm{FBS}$ or conditioned medium. After $4 \mathrm{~h}$, the migrated cells were stained with $0.1 \%$ crystal violet and photographed by phase contrast microscopy ( $4 \times$ ) in three individual fields. (A) Migration of MSC and MSC/Fluc cells. (B) Migration of MSC/Fluc cells with CAL62 and MDA-MB-231 conditioned medium. Data from three independent results were expressed as the means \pm standard deviation (SD), and a $p$-value $<0.05$ was considered significant, according to the Student's $t$-test. *** represents the significance between the SFM and conditioned medium respect with CAL62 and MDA-MB-231/Rluc. \# represents the significance between CAL62/Rluc and MDA-MB-231 at the level of $p<0.05$.

\section{Therapeutic effect of MSCs}

To confirm the drug delivery-based therapeutic effect of MSCs, here we used DOX for the loading and confirmation of drug delivery, owing to its fluorescence properties. CAL62/Rluc cells and MDA-MB-231/Rluc cells after co-culture with DOX-pretreated MSCs for 24 and $48 \mathrm{~h}$ (Figure 3A and 3B). Rluc activity of MDA-MB-231/Rluc ( $<<0.01$ and $\mathrm{p}<0.001)$ and CAL62/Rluc $(\mathrm{p}<0.05)$ differed significantly with increased ratios (1:0.5, 1:1, 1:2) of DOX-pretreated MSCs at $24 \mathrm{~h}$, compared than with naïve MSCs. Additionally, 48-h Rluc activity was also significantly reduced in both MDA-MB-231/Rluc $(p<0.01$ and $p<0.001)$ and CAL62/Rluc $(p<0.01$ and $p<0.001)$. Also, we confirmed DOX fluorescence in DOX-pretreated MSCs by confocal microscopy
(Suppl. Figure 1). CAL62/Rluc and MDA-MB-231/ Rluc cells showed DOX fluorescence after a 24-h co-culture with DOX-pretreated MSCs, but DOX fluorescence was not seen with naïve MSCs (Suppl. Figure 2 and 3). These results confirm that MSCs can be used as delivery vehicles of anticancer agents.

\section{Effect of DOX on MSC}

To confirm the DOX effect on MSCs, we treated different concentrations of DOX for $12 \mathrm{~h}$. We found that DOX did not affect the cell viability of MSCs at 5 $\mu \mathrm{M}$ concentration (Suppl Fig 4), but decreased cell viability with higher concentrations of DOX at 10 and $20 \mu \mathrm{M}(\mathrm{p}<0.05)$. Therefore, DOX at $5 \mu \mathrm{M}$ concentration was not interference of MSC viability, could be used for loading to MSCs. 
A
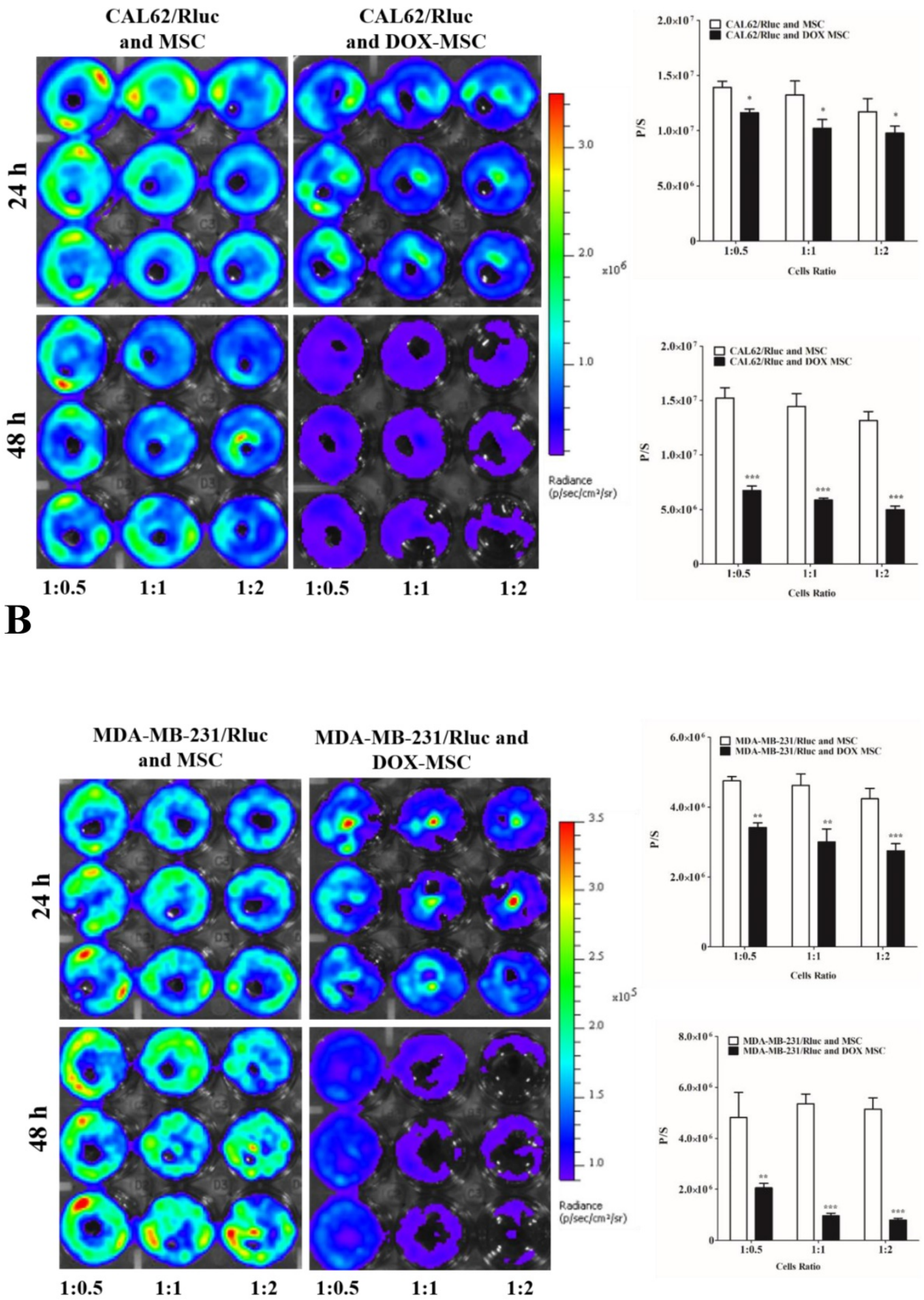

Figure 3. Rluc activity of cancer cells. The MDA-MB-231/Rluc and CAL62/Rluc cells were co-cultured with different ratios of Doxorubicin-pretreated MSCs with, and the Rluc activity of cancer cells was measured 24 and $48 \mathrm{~h}$ after the co-culturing. A) Rluc activity of MDA-MB-231/Rluc. B) Rluc activity of CAL62/Rluc. Data were expressed as the means \pm standard deviation $(\mathrm{SD})$, and a $p$-value $<0.05$ was considered significant, according to the Student's $t$-test.

\section{In vivo migration of MSC/Fluc}

To track the MSC/Fluc in inflamed microenvironment, we developed MDA-MB-231/ Rluc and CAL62/Rluc xenograft models in nude mice, the tumor xenografts were detected by the bioluminescent imaging of Rluc activity by using the IVIS system (Figure 4A and Figure 5A). The established xenograft models were systemically injected $1 \times 10^{6}$ 
MSC/Fluc. Mice were imaged at $1 \mathrm{~h}$ and $24 \mathrm{~h}$ by noninvasive BLI after five minutes injection of D-Luciferin. BLI signals associated with MSC/Fluc cells were highly detected in the lung area 1 and $24 \mathrm{~h}$ after injection, as most i.v.-injected cells were trapped mainly in the lungs and also Fluc activity seen in other parts of organs such as liver and bone marrow. Tumor region Fluc activity was measured by creating ROI over the tumor area and measured. The Fluc signal intensity of MSC/Fluc at the CAL62 tumor area $(\mathrm{p}<0.05)$ and MDA-MB-231 tumor area $(\mathrm{p}<0.05)$ was significantly higher than that in the control group (Figure 4B and 5B). Fluc activity of the ex vivo tumor confirmed the migration of MSC/Fluc to both tumors (Figure $4 \mathrm{C}$ and $5 \mathrm{C}$ ). We further confirmed the GFP positive MSC/Fluc cells in the MDA-MB-231 tumor by immunohistochemistry (Fig 5D). In the both tumors MSC/Fluc migrated cells were less in activity it may be the passage number dependent and or other migratory factors decreased in MSCs.

\section{Discussion}

In this study, we confirmed that after intravenous systemic injection, MSCs migrate to breast and thyroid tumors in in vivo animal models. The tumor tropism of MSCs has gained attention owing to their potential to be used as drug delivery vehicles for cancer treatment. Studies suggested that MSCs can be used as drug delivery agents; for example, interferon $\beta$ and tumor necrosis factor (TNF) genes inhibited tumor progression [29, 32]. MSCs were isolated from human bone marrow, cultured for 5-9 passages, and used for systemic injection. MSCs were trapped in lungs or lymph nodes and disappeared after cell injection. However, the mechanism and behavior of MSCs was not clear, as they secrete various bioactive molecules under different conditions [33, 34]. Between these molecules, several diverse stimulatory factors such as interleukin-6, TNFa, and SDF1 interacted with MSCs $[33,35-37]$. This interaction occurs because of the presence of receptors on the membrane of MSCs [38];
A

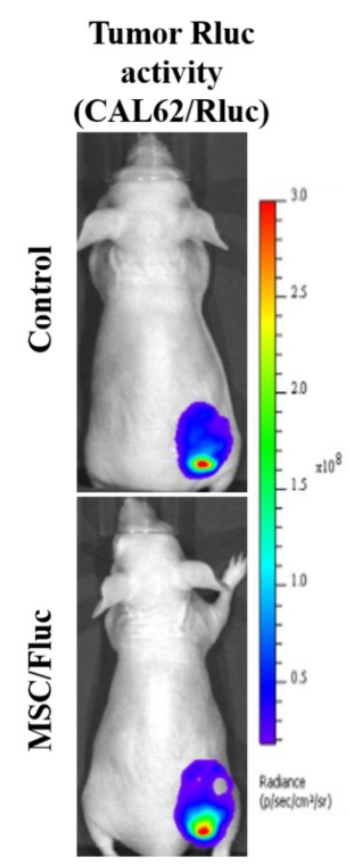

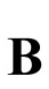
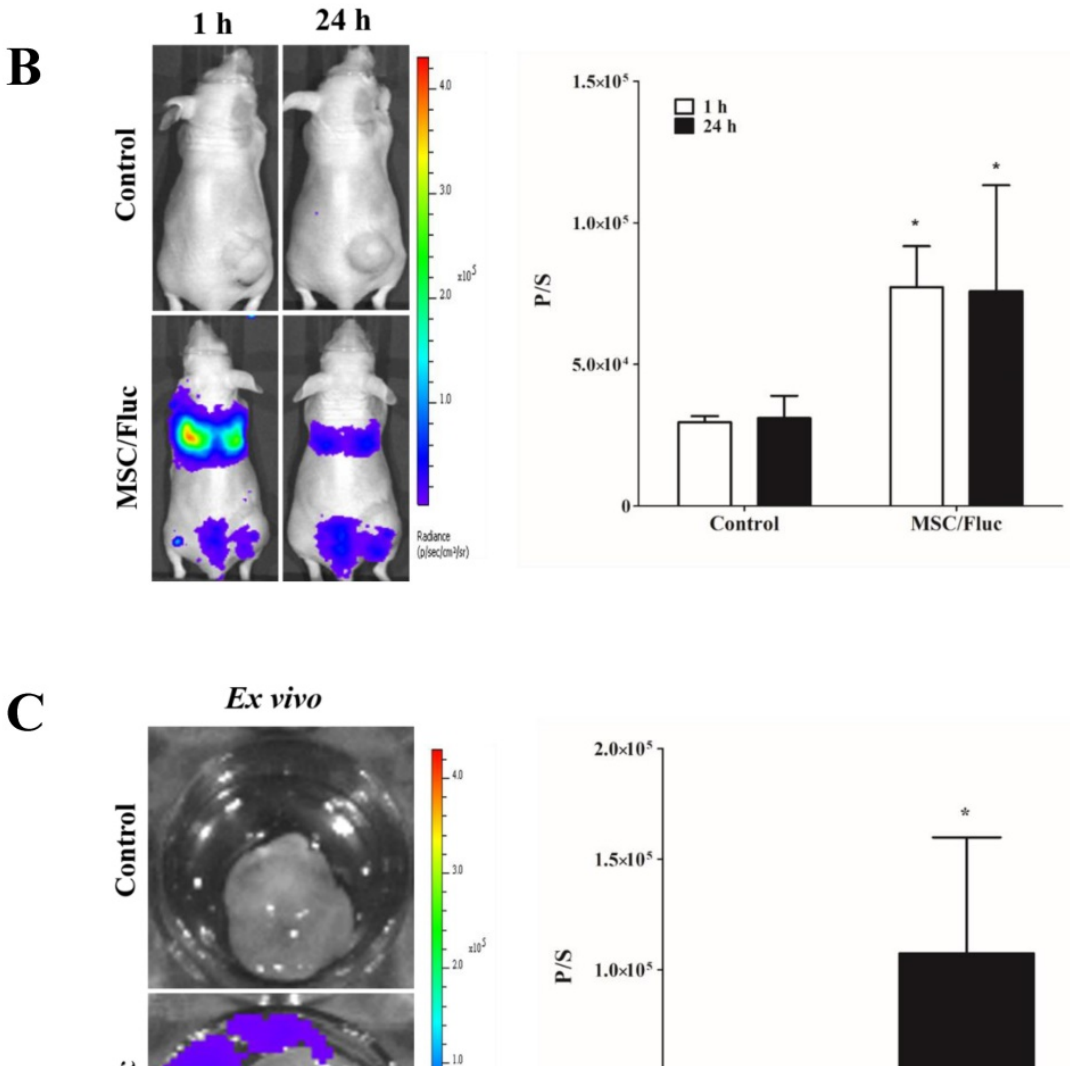
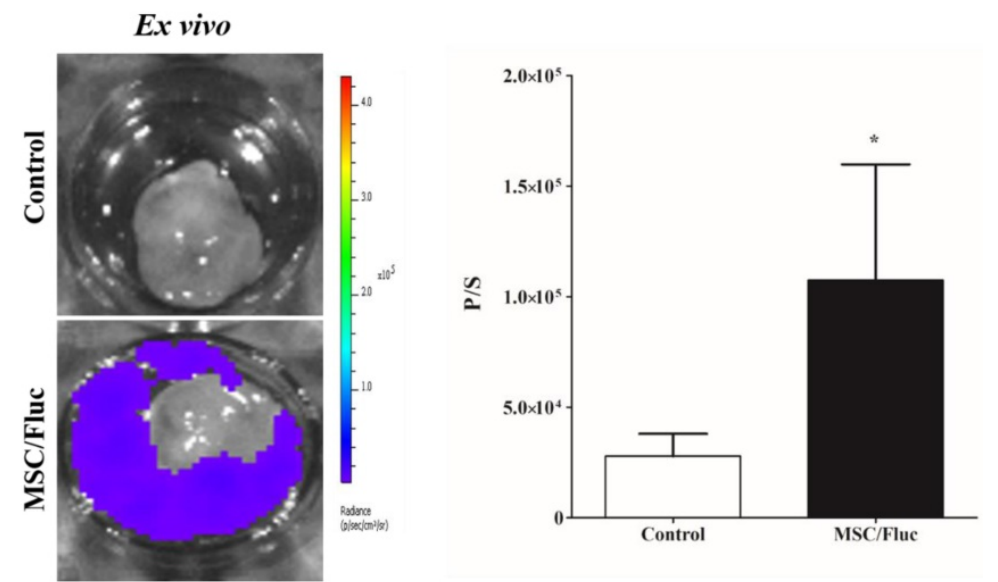

Figure 4. In vivo migration of MSC/Fluc to CAL62/Rluc tumor. (A) Rluc activity of anaplastic thyroid (CAL62) tumor, (B) MSC/Fluc cells were systemically injected into mouse bearing the CAL62/Rluc xenograft tumor, while PBS was injected as a control. The Fluc activity of MSC/Fluc cells was measured 1 and $24 \mathrm{~h}$ after the injection, and (C) ex vivo Fluc activity of MSC/Fluc cells was measured using CAL62 tumor cells. Quantitative analysis of the BLI signals were measured in three mice, and the data were expressed as the means \pm standard deviation (SD). A p-value $<0.05$ was considered significant, according to the Student's $t$-test. 


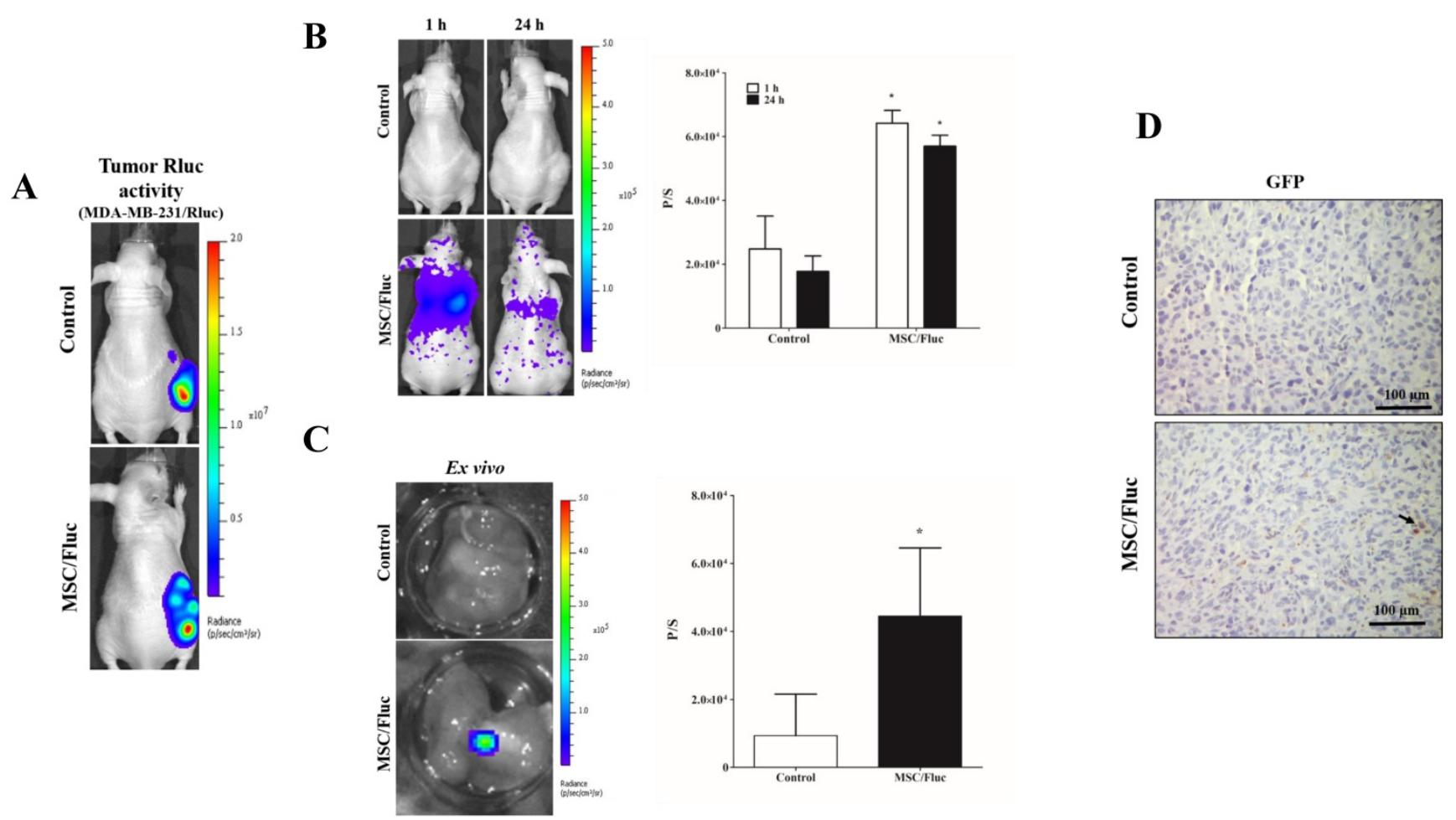

Figure 5. In vivo migration of the MSC/Fluc cells towards the MDA-MB-231/Rluc tumor. (A) Rluc activity of the MDA-MB-231 tumor, (B) MSC/Fluc cells were systemically injected into mice bearing the MDA-MB-231/Rluc xenograft tumor, while PBS was injected as a control. The Fluc activity of MSC/Fluc cells was measured 1 and $24 \mathrm{~h}$ after the injection, and (C) ex vivo Fluc activity of MSC/Fluc was measured using MDA-MB-231 tumor cells. Quantitative analysis of the BLI signals were measured in three mice, and the data were expressed as the means \pm standard deviation (SD). (D) Immunohistochemistry analysis MSC/Fluc cells with the GFP specific antibody in the excised tumor. With a $p$-value $<0.05$ was considered significant by Student's $t$-test.

these stimulatory factors are strongly involved during the migration process [33]. In order to confirm the migration of MSCs, we successfully generated MSC/Fluc cells. First, we confirmed the functional efficiency by an in vitro migration assay; MSCs and MSC/Fluc cells migrated towards the chemoattractant-containing medium (0.5\% FBS) after a 4-h incubation (Figure 2). These in vitro results confirm that transduction does not influence the migration potency of the MSCs. We also confirmed conditioned medium of cancer cells dependent migration as well as DOX treated MSCs also migrated with slight decreased in numbers nut not significantly.

MSCs can proliferate in culture with consistent morphology, surface marker proteins, and differentiation potential for multiple mesenchymal lineages under in vitro conditions. The precise evaluation and assessment of survival, engraftment, and fate of MSCs in a surrogate animal model after their systemic administration are essential for developing MSCbased cell therapies [3]. Here, we used human breast cancer and thyroid cancer tumor models, in which tumor cells were transduced with Rluc (MDA-MB231/Rluc and CAL62/Rluc) and subjected to MSCs expressing Fluc (MSC/Fluc). Bi-reporter gene-based cell labeling was used to simultaneously detect both the xenograft tumors and injected MSCs in each mouse.
MSCs released from the bone marrow migrate to inflamed tissues and are disturbed by direct contact or in a paracrine manner in response to inflammatory cells such as dendritic cells [39], macrophages [40], and T-cells [41]. Studies have focused on the effects of stem cell migration and engraftment to disease sites. MSC migration based on molecular signaling cascades are particularly important, as the Wnt signaling pathway is related to migration and invasion [42]. MSC migration involves numerous growth factors (GFs). One important GF for epithelial cell and MSC migration is the hepatocyte growth factor (HGF). MSCs constitutively express the HGF ligand c-met in response to HGF-dependent migration [43]. Although the tissue homing capability of MSCs is associated with CXCR12, CXCR4, and CCL2, CCL2 has been implicated in the tissue-homing ability of MSCs [44], but the precise mechanism of MSC migration towards tumors remains unclear.

MSCs have been reported to promote cancer progression by immune modulation [45]; however, other studies also revealed an inhibitory effect of MSCs on the development of tumors, via the modification of Akt signaling [46]. These inconsistent results may be related to the use of cells from different tissue sources, donor variability between individuals, and the timing of MSC injections. MSCs can suppress or support tumor growth and be recruited or migrate 
towards tumor sites when administered systemically $[15,29]$. These findings suggest that targeted drug therapy can be developed for cancer by utilizing engineered MSCs. In the present study, we successfully developed MSCs expressing Fluc, and visualized the migration of MSCs towards breast and anaplastic thyroid cancer cells in vivo and ex vivo by optical imaging (Figure 4C and 5C). This MSC tropism to various tumors supports the value of using exogenous MSCs as biological carriers for cancerous diseases. Administered allogenic MSCs were observed in the lungs, and then in the spleen and liver of SCID mice [1, 47].

MSC migration to inflammatory state offers many therapeutic strategies including cancer $[12,48]$. Levels of stromal cell-derived factor (SDF-1) and CXCR4 are higher in injured or stressed tissues [49, 50], this ligand/ receptor pair may facilitate the migration of stem cells into damaged areas of the tissues [51, 52]. Tumor cells secrete chemokines, which recruit circulating MSCs, through the SDF-1a/CXCR4 pathway $[53,54]$. In the current study, the migration of MSC/Fluc cells to the tumor sites was confirmed $24 \mathrm{~h}$ after the systemic injection of the cells by both in vivo and ex vivo BLI in mouse models. Kyriakou et al., 2008 reported that short term in vivo migration of fluorescence stained hMSCs decreased $8-12^{\text {th }}$ passage to other organs such as bone marrow and spleen [55]. Consistent with above study another report from Rombus et al., 2003 found that the homing efficiency of MSCs decreased with extended ex vivo culture of MSCs isolated from human bone marrow was confirmed with immunodeficient xenogeneic model [56]. In this study Fluc activity of the migrated MSC/Fluc cells was less this in supports from above studies it may be the reason for the less migration potential. Kidd et al., reported that the hMSC biodistribution in inflamed microenvironment in SCID mice with the generated cutaneous wounds model with i.v injected MSCs started to migrate at the site after 3 days and stay at the inflamed region. They also speculate that initial decline of photon flux because of loss of MSC which may fail to stimulate critical survival or adhesion processes [1]. In our study low number of MSCs was targeted to tumor, which may be increased by overexpression of chemokine receptors or other tumor targeting factors in MSCs, such enhanced migration of MSCs to target tumor can provide a better therapeutic effect. Since, it was reported that CXCR4 overexpression increased the in vivo migration ability of the MSCs to tumor [30, 57].

Since MSCs easily adapt to culture conditions and home to pathological tissues when injected into in vivo models, they seem to be a good choice for delivering anticancer agents [58]. Therefore, in this study, we confirmed that the MSCs can serve as drug delivery vehicles, when DOX-pretreated MSCs killed the cancer cells (Figure 3A and 3B) due to transfer of DOX in the CAL62/Rluc and MDA-MB-231/Rluc cells, as confirmed by confocal microscopy (Suppl. Figure 2 and 3). Previous studies also demonstrated that MSCs were loaded with the anticancer drug paclitaxel in vitro, and loaded MSCs were used for cancer treatment in vivo [26].

Recently, Zhao et al., reported targeted delivery of DOX using MSCs to lung melanoma metastasis. They successfully loaded nano-DOX in MSCs and confirmed therapeutic effect of the MSCs [59]. Therefore, delivery of DOX or Nano-DOX using MSCs may be possible as a targeted drug delivery strategy. Based on their tumor targeting ability and feasible DOX loading, MSCs may be used as a source of cell-based therapies against intractable breast and thyroid cancers. However, further studies are required to develop an ideal MSC-based cancer therapy, by selecting the appropriate dose of DOX or nano DOX for achieving optimal loading of DOX onto MSCs, and modulating MSCs to enhance their tumor-targeting ability.

\section{Conclusion}

This study showed that Fluc transduced bone marrow-derived MSCs, and the MSCs migrated towards the breast and anaplastic thyroid cancer cells. DOX-pretreated MSCs deliver DOX to cancer cells. Therefore, MSCs may be used for cell therapy in preclinical settings, and that their in vivo activity should be evaluated. After confirmative therapy, they may be used for clinical trials.

\section{Ethics Statement}

Animal experiments were approved by the Institutional Animal Care and Use Committee (KNU2012-43) of The Kyungpook National University of Korea.

\section{Acknowledgments}

This research was supported by: a Basic Science Research Program through the National Research Foundation of Korea (NRF) funded by the Ministry of Education (NRF-2016R1D1A1A02936968); a National Research Foundation of Korea (NRF) grant funded by the Korea government (MSIP) (no. NRF-2015M2A2 A7A01045177); and a grant from the Korea Health Technology R\&D Project, Ministry of Health \& Welfare, Republic of Korea (HI16C1501). 


\section{Supplementary Material}

Supplementary figures.

http://www.medsci.org/v15p1051s1.pdf

\section{Competing Interests}

The authors have declared that no competing interest exists.

\section{References}

1. Kidd S, Spaeth E, Dembinski JL, Dietrich M, Watson K, Klopp A, et al. Direct evidence of mesenchymal stem cell tropism for tumor and wounding microenvironments using in vivo bioluminescent imaging. Stem Cells. 2009; 27: 2614-23.

2. Cao J, Hou S, Ding H, Liu Z, Song M, Qin X, et al. In Vivo Tracking of Systemically Administered Allogeneic Bone Marrow Mesenchymal Stem Cells in Normal Rats through Bioluminescence Imaging. Stem Cells International. 2016; 2016.

3. Wang H, Cao F, De A, Cao Y, Contag C, Gambhir SS, et al. Trafficking mesenchymal stem cell engraftment and differentiation in tumor-bearing mice by bioluminescence imaging. Stem Cells. 2009; 27: 1548-58.

4. Reagan MR, Kaplan DL. Concise Review: Mesenchymal Stem Cell Tumor-Homing: Detection Methods in Disease Model Systems. Stem Cells. 2011; 29: 920-7.

5. Dasari VR, Veeravalli KK, Dinh DH. Mesenchymal stem cells in the treatment of spinal cord injuries: a review. World J Stem Cells. 2014; 6: 120-33.

6. Liu J, Han D, Wang Z, Xue M, Zhu L, Yan H, et al. Clinical analysis of the treatment of spinal cord injury with umbilical cord mesenchymal stem cells. Cytotherapy. 2013; 15: 185-91.

7. Dixit P, Katare R. Challenges in identifying the best source of stem cells for cardiac regeneration therapy. Stem cell research \& therapy. 2015; 6: 26.

8. Markert CD, Atala A, Cann JK, Christ G, Furth M, Ambrosio F, et al. Mesenchymal stem cells: emerging therapy for Duchenne muscular dystrophy. PM\&R. 2009; 1: 547-59.

9. Ko I-K, Kim B-S. Mesenchymal stem cells for treatment of myocardial infarction. International journal of stem cells. 2008; $1: 49$.

10. Satessa G, Lenjisa J, Gebremariam E, Woldu M. Stem cell therapy for myocardial infarction: challenges and prospects. J Stem Cell Res Ther. 2015; 5: 2.

11. Dunavin N, Dias A, Li M, McGuirk J. Mesenchymal Stromal Cells: What Is the Mechanism in Acute Graft-Versus-Host Disease? Biomedicines. 2017; 5: 39

12. Nakamizo A, Marini F, Amano T, Khan A, Studeny M, Gumin J, et al. Human bone marrow-derived mesenchymal stem cells in the treatment of gliomas. Cancer Res. 2005: 65: 3307-18.

13. Ren C, Kumar S, Chanda D, Kallman L, Chen J, Mountz JD, et al. Cancer gene therapy using mesenchymal stem cells expressing interferon- $\beta$ in a mouse prostate cancer lung metastasis model. Gene Ther. 2008; 15: 1446.

14. Duan X, Guan H, Cao Y, Kleinerman ES. Murine bone marrow-derived mesenchymal stem cells as vehicles for interleukin-12 gene delivery into Ewing sarcoma tumors. Cancer. 2009; 115: 13-22

15. Studeny M, Marini FC, Champlin RE, Zompetta C, Fidler IJ, Andreeff M. Bone marrow-derived mesenchymal stem cells as vehicles for interferon- $\beta$ delivery into tumors. Cancer Res. 2002; 62: 3603-8.

16. Klopp AH, Spaeth EL, Dembinski JL, Woodward WA, Munshi A, Meyn RE, et al. Tumor irradiation increases the recruitment of circulating mesenchymal stem cells into the tumor microenvironment. Cancer Res. 2007; 67: 11687-95.

17. Pereira R, Halford K, O'hara M, Leeper D, Sokolov B, Pollard M, et al. Cultured adherent cells from marrow can serve as long-lasting precursor cells for bone, cartilage, and lung in irradiated mice. Proceedings of the national academy of sciences. 1995; 92: 4857-61.

18. Allers C, Sierralta WD, Neubauer S, Rivera F, Minguell JJ, Conget PA. Dynamic of distribution of human bone marrow-derived mesenchymal stem cells after transplantation into adult unconditioned mice. Transplantation. 2004; 78 : 503-8.

19. Ahn BC. Nuclear Medicine in the Era of Precision Medicine. Nucl Med Mol Imaging. 2017. p. 99-100.doi: 10.1007/s13139-017-0478-5.

20. Lee HW, Gangadaran P, Kalimuthu S, Ahn B-C. Advances in molecular imaging strategies for in vivo tracking of immune cells. BioMed research international. 2016; 2016

21. Ahn B-C. Requisites for successful theranostics with radionuclide-based reporter gene imaging. J Drug Target. 2014; 22: 295-303.

22. Krishnan M, Park JM, Cao F, Wang D, Paulmurugan R, Tseng JR, et al. Effects of epigenetic modulation on reporter gene expression: implications for stem cell imaging. The FASEB journal. 2006; 20: 106-8.

23. Kalimuthu S, Oh JM, Gangadaran P, Zhu L, Lee HW, Rajendran RL, et al. In Vivo Tracking of Chemokine Receptor CXCR4-Engineered Mesenchymal Stem Cell Migration by Optical Molecular Imaging. Stem cells international. 2017; 2017.
24. Kalimuthu S, Jeong JH, Oh JM, Ahn B-C. Drug Discovery by Molecular Imaging and Monitoring Therapy Response in Lymphoma. Int J Mol Sci. 2017; 18: 1639.

25. Kim JE, Kalimuthu S, Ahn B-C. In Vivo Cell Tracking with Bioluminescence Imaging. Nucl Med Mol Imag. 2015; 49: 3-10.

26. Pessina A, Bonomi A, Coccè V, Invernici G, Navone S, Cavicchini L, et al. Mesenchymal stromal cells primed with paclitaxel provide a new approach for cancer therapy. PLoS One. 2011; 6: e28321.

27. Weldon JE, Xiang L, Chertov O, Margulies I, Kreitman RJ, FitzGerald DJ, et al. A protease-resistant immunotoxin against CD22 with greatly increased activity against CLL and diminished animal toxicity. Blood. 2009; 113: 3792-800.

28. Dhar S, Gu FX, Langer R, Farokhzad OC, Lippard SJ. Targeted delivery of cisplatin to prostate cancer cells by aptamer functionalized Pt (IV) prodrug-PLGA-PEG nanoparticles. Proceedings of the National Academy of Sciences. 2008; 105: 17356-61.

29. Loebinger MR, Eddaoudi A, Davies D, Janes SM. Mesenchymal stem cell delivery of TRAIL can eliminate metastatic cancer. Cancer Res. 2009; 69: 4134-42

30. Kalimuthu S, Oh JM, Gangadaran P, Zhu L, Lee HW, Jeon YH, et al. Genetically engineered suicide gene in mesenchymal stem cells using a Tet-On system for anaplastic thyroid cancer. PLoS One. 2017; 12.

31. Kalimuthu S, Oh JM, Gangadaran P, Zhu L, Lee HW, Rajendran RL, et al. In Vivo Tracking of Chemokine Receptor CXCR4-Engineered Mesenchymal Stem Cell Migration by Optical Molecular Imaging. Stem Cells International. 2017; 2017: https://doi.org/10.1155/2017/8085637.

32. Grisendi G, Bussolari R, Cafarelli L, Petak I, Rasini V, Veronesi E, et al. Adipose-derived mesenchymal stem cells as stable source of tumor necrosis factor-related apoptosis-inducing ligand delivery for cancer therapy. Cancer Res. 2010; 70: 3718-29.

33. Yagi $\mathrm{H}$, Soto-Gutierrez A, Navarro-Alvarez $\mathrm{N}$, Nahmias $\mathrm{Y}$, Goldwasser $\mathrm{Y}$ Kitagawa $Y$, et al. Reactive bone marrow stromal cells attenuate systemic inflammation via sTNFR1. Mol Ther. 2010; 18: 1857-64.

34. Crisostomo PR, Wang M, Herring CM, Markel TA, Meldrum KK, Lillemoe $\mathrm{KD}$, et al. Gender differences in injury induced mesenchymal stem cell apoptosis and VEGF, TNF, IL-6 expression: role of the $55 \mathrm{kDa}$ TNF receptor (TNFR1). J Mol Cell Cardiol. 2007; 42: 142-9.

35. Ceradini DJ, Kulkarni AR, Callaghan MJ, Tepper OM, Bastidas N, Kleinman $\mathrm{ME}$, et al. Progenitor cell trafficking is regulated by hypoxic gradients through HIF-1 induction of SDF-1. Nat Med. 2004; 10: 858

36. English K, Barry FP, Field-Corbett CP, Mahon BP. IFN- $\gamma$ and TNF-a differentially regulate immunomodulation by murine mesenchymal stem cells. Immunol Lett. 2007; 110: 91-100.

37. Markel TA, Crisostomo PR, Wang M, Herring CM, Meldrum DR. Activation of individual tumor necrosis factor receptors differentially affects stem cell growth factor and cytokine production. American Journal of Physiology-Gastrointestinal and Liver Physiology. 2007; 293: G657-G62.

38. Ip JE, Wu Y, Huang J, Zhang L, Pratt RE, Dzau VJ. Mesenchymal stem cells use integrin $\beta 1$ not CXC chemokine receptor 4 for myocardial migration and engraftment. Mol Biol Cell. 2007; 18: 2873-82.

39. Djouad F, Charbonnier LM, Bouffi C, Louis-Plence P, Bony C, Apparailly F, et al. Mesenchymal stem cells inhibit the differentiation of dendritic cells through an interleukin-6-dependent mechanism. Stem Cells. 2007; 25: 2025-32.

40. Ehninger A, Trumpp A. The bone marrow stem cell niche grows up: mesenchymal stem cells and macrophages move in. J Exp Med. 2011; 208: 421-8.

41. Glennie S, Soeiro I, Dyson PJ, Lam EW-F, Dazzi F. Bone marrow mesenchymal stem cells induce division arrest anergy of activated T cells. Blood. 2005; 105: $2821-7$.

42. Neth P, Ciccarella M, Egea V, Hoelters J, Jochum M, Ries C. Wnt signaling regulates the invasion capacity of human mesenchymal stem cells. Stem Cells. 2006; 24: 1892-903.

43. Neuss S, Becher E, Wöltje M, Tietze L, Jahnen-Dechent W. Functional expression of HGF and HGF receptor/c-met in adult human mesenchymal stem cells suggests a role in cell mobilization, tissue repair, and wound healing. Stem Cells. 2004; 22: 405-14.

44. Dwyer R, Potter-Beirne S, Harrington K, Lowery A, Hennessy E, Murphy J, et al. Monocyte chemotactic protein-1 secreted by primary breast tumors stimulates migration of mesenchymal stem cells. Clin Cancer Res. 2007; 13: 5020-7.

45. Jo YJ. Mesenchymal Stem Cells within Tumor Stroma Promote Breast Cancer Metastasis. The Korean Journal of Gastroenterology. 2007; 50: 344-5.

46. Khakoo AY, Pati S, Anderson SA, Reid W, Elshal MF, Rovira II, et al. Human mesenchymal stem cells exert potent antitumorigenic effects in a model of Kaposi's sarcoma. J Exp Med. 2006; 203: 1235-47.

47. Albarenque SM, Zwacka RM, Mohr A. Both human and mouse mesenchymal stem cells promote breast cancer metastasis. Stem Cell Res. 2011; 7: 163-71.

48. Studeny M, Marini FC, Dembinski JL, Zompetta C, Cabreira-Hansen M, Bekele BN, et al. Mesenchymal stem cells: potential precursors for tumor stroma and targeted-delivery vehicles for anticancer agents. J Natl Cancer Inst. 2004; 96: 1593-603.

49. Schioppa T, Uranchimeg B, Saccani A, Biswas SK, Doni A, Rapisarda A, et al. Regulation of the chemokine receptor CXCR4 by hypoxia. J Exp Med. 2003; 198: 1391-402. 
50. Ponomaryov T, Peled A, Petit I, Taichman RS, Habler L, Sandbank J, et al. Induction of the chemokine stromal-derived factor-1 following DNA damage improves human stem cell function. The Journal of clinical investigation. 2000; 106: 1331-9.

51. Yamaguchi J-i, Kusano KF, Masuo O, Kawamoto A, Silver M, Murasawa S, et al. Stromal cell-derived factor-1 effects on ex vivo expanded endothelial progenitor cell recruitment for ischemic neovascularization. Circulation. 2003; 107: 1322-8.

52. Askari AT, Unzek S, Popovic ZB, Goldman CK, Forudi F, Kiedrowski M, et al. Effect of stromal-cell-derived factor 1 on stem-cell homing and tissue regeneration in ischaemic cardiomyopathy. The Lancet. 2003; 362: 697-703.

53. Quan C, Cho MK, Shao Y, Mianecki LE, Liao E, Perry D, et al. Dermal fibroblast expression of stromal cell-derived factor-1 (SDF-1) promotes epidermal keratinocyte proliferation in normal and diseased skin. Protein Cell. 2015; 6: 890-903.

54. Zhou SB, Wang J, Chiang CA, Sheng LL, Li QF. Mechanical Stretch Upregulates SDF-1a in Skin Tissue and Induces Migration of Circulating Bone Marrow-Derived Stem Cells into the Expanded Skin. Stem Cells. 2013; 31: 2703-13.

55. Kyriakou C, Rabin N, Pizzey A, Nathwani A, Yong K. Factors that influence short-term homing of human bone marrow-derived mesenchymal stem cells in a xenogeneic animal model. Haematologica. 2008; 93: 1457-65.

56. Rombouts W, Ploemacher R. Primary murine MSC show highly efficient homing to the bone marrow but lose homing ability following culture. Leukemia. 2003; 17: 160.

57. Park SA, Ryu CH, Kim SM, Lim JY, Park SI, Jeong $\mathrm{CH}$, et al. CXCR4-transfected human umbilical cord blood-derived mesenchymal stem cells exhibit enhanced migratory capacity toward gliomas. Int J Oncol. 2011; 38: 97-103.

58. Menon LG, Shi VJ, Carroll RS. Mesenchymal stromal cells as a drug delivery system. 2009.

59. Zhao Y, Tang S, Guo J, Alahdal M, Cao S, Yang Z, et al. Targeted delivery of doxorubicin by nano-loaded mesenchymal stem cells for lung melanoma metastases therapy. Scientific reports. 2017; 7: 44758. 